\title{
RESEARCH HIGHLIGHT SARS-CoV-2-induced lung pathology: AHR as a candidate therapeutic target
}

\author{
Federico Giovannoni ${ }^{1}$ and Francisco J. Quintana $\mathbb{D}^{1,2}$ \\ Cell Research (2021) 31:1-2; https://doi.org/10.1038/s41422-020-00447-9
}

\begin{abstract}
The aryl hydrocarbon receptor (AHR) is activated by multiple viruses to evade the host immune response, a strategy exploited in pre-clinical models to limit the replication of Zika and Influenza A. In a recent study, Liu et al. report that AHR drives the hypersecretion of lung mucins after SARS-CoV2 infection, suggesting a role for AHR in respiratory failure and highlighting its potential therapeutic value.
\end{abstract}

COVID-19 shows a wide spectrum of clinical severity, ranging from asymptomatic or mild infection ( $80 \%$ of cases) to severe and critical life-threatening forms of the disease ( 5\%-15\%). The primary cause of death in severe COVID-19 patients is progressive respiratory failure. Since respiratory symptoms in these patients usually worsen a week after disease onset, it has been suggested that they result from a dysregulated pro-inflammatory response, which eventually damages lung epithelial and endothelial cells, impairing the exchange of $\mathrm{O}_{2}$ and $\mathrm{CO}_{2}{ }^{1}$. An imbalanced inflammatory response, however, does not explain hypoxia in all COVID-19 patients. Indeed, severe hypoxia has also been reported at early stages of COVID-19, before an excessive inflammatory response is established. Intriguingly, despite presenting low blood $\mathrm{O}_{2}$ levels, some of these patients show minimal symptoms and apparent distress, a condition referred to as 'silent hypoxia'. ${ }^{2}$ The mechanism responsible for the development of silent hypoxia is still lacking. In a recent work published in Cell Research, Liu et al. report that SARS-CoV-2-triggered IFN signaling induces mucin overproduction by lung epithelial cells, thickening the blood-air barrier and hindering $\mathrm{O}_{2}$ diffusion, leading to hypoxia. ${ }^{3}$ Moreover, they show that mucin expression is driven by the transcription factor aryl hydrocarbon receptor (AHR), identifying AHR as a potential target for the treatment of hypoxia in COVID-19 patients.

Liu et al. first detected increased expression of mucins in bronchoalveolar lavage (BALF) samples taken from COVID-19 patients and macaques infected with SARS-CoV-2, in agreement with independent scRNA-Seq studies ${ }^{4}$ and the detection of increased mucin expression and mucus production in COVID-19 autopsy samples. ${ }^{5}$ Mucus hypersecretion in COVID-19 patients has been associated with airflow obstruction and respiratory distress, hence the mechanisms that control it are considered therapeutic targets of interest. Through a combination of in vitro and in vivo experiments, Liu et al. found that IFN- $\beta$ and IFN- $\gamma$ upregulate mucin production in lung epithelial cells. IFNs are known to activate AHR signaling, e.g., by inducing the expression of the enzymes IDO1/TDO2 which catalyze the generation of the AHR agonist Kynurenine (Kyn) ${ }^{6,7}$ Indeed, the authors found that an IFNIDO-Kyn-AHR axis drives mucin expression in lung epithelial cells.
Finally, the authors used a murine model to evaluate the translational implications of their work. Using human ACE2 transgenic mice, they found that SARS-CoV-2 induced the upregulation of lung mucin expression and decrease in $\mathrm{O}_{2}$ levels in peripheral blood, which was reverted by the administration of an AHR antagonist, identifying AHR as a candidate target to treat SARS-CoV-2-induced lung pathology.

AHR signaling has been shown to play a physiological role in the regulation of the host anti-viral response. ${ }^{8-10}$ Type I IFN (IFN-I), the central regulator of the anti-viral response, induces AHR expression, but AHR can suppress the expression of IFN-I, most likely as part of a negative feedback loop. ${ }^{6-9}$ Moreover, AHR has also been shown to inhibit NF-KB, an additional key effector molecule in the host anti-viral and inflammatory response. ${ }^{6,7,9}$ Previous studies using AHR antagonists and gene knockdown have shown that AHR inactivation reduces Influenza A, Zika and Dengue virus replication. 8 . 9 These findings led to the hypothesis that AHR is a pro-viral host factor targeted by multiple viruses to limit IFN-I/NF-KB-driven host anti-viral immunity and promote virus replication (Fig. 1). The identification of AHR as a pro-viral host factor also has important therapeutic implications. Indeed, in mice infected with Influenza A virus, AHR antagonism increased IFN- $\beta$ levels, reduced BALF viral titers and increased survival. ${ }^{8}$ AHR antagonism also reduced Zika virus replication in fetuses and ameliorated congenital Zika virus syndrome in a pre-clinical mouse model. ${ }^{9}$

It was recently reported that infection with human coronaviruses, including SARS-CoV-2, activated AHR signaling, as determined by the RNA-seq analysis of lung epithelial cells. ${ }^{10}$ This finding triggered the question of whether AHR also plays a role as a pro-viral host factor in the replication of coronaviruses and, consequently, can be a candidate therapeutic target against SARS-CoV-2. The work by Liu et al. uncovers an additional benefit of targeting AHR during SARSCoV-2 infection; pharmacologic inhibition of AHR may not only boost anti-viral immunity, but also directly suppress mechanisms of lung pathology (Fig. 1). However, since the effects of AHR inhibition on lung SARS-CoV-2 replication were not assessed, Liu et al. cannot rule out the possibility that the reduction in virus-induced lung pathology results from the suppression of SARS-CoV-2 replication. AHR antagonists likely ameliorate lung pathology by both boosting anti-viral immunity and limiting virus replication, and also by suppressing excessive mucus production.

Independently of the specific mechanisms involved in the therapeutic effects of AHR antagonists on SARS-CoV-2 infection, the last few years have seen an increasing number of reports

\footnotetext{
${ }^{1}$ Ann Romney Center for Neurologic Diseases, Brigham and Women's Hospital, Harvard Medical School, Boston, MA 02115, USA and ${ }^{2}$ Broad Institute of MIT and Harvard, Cambridge, MA, USA

Correspondence: Francisco J. Quintana (fquintana@rics.bwh.harvard.edu)
}

Published online: 1 December 2020 


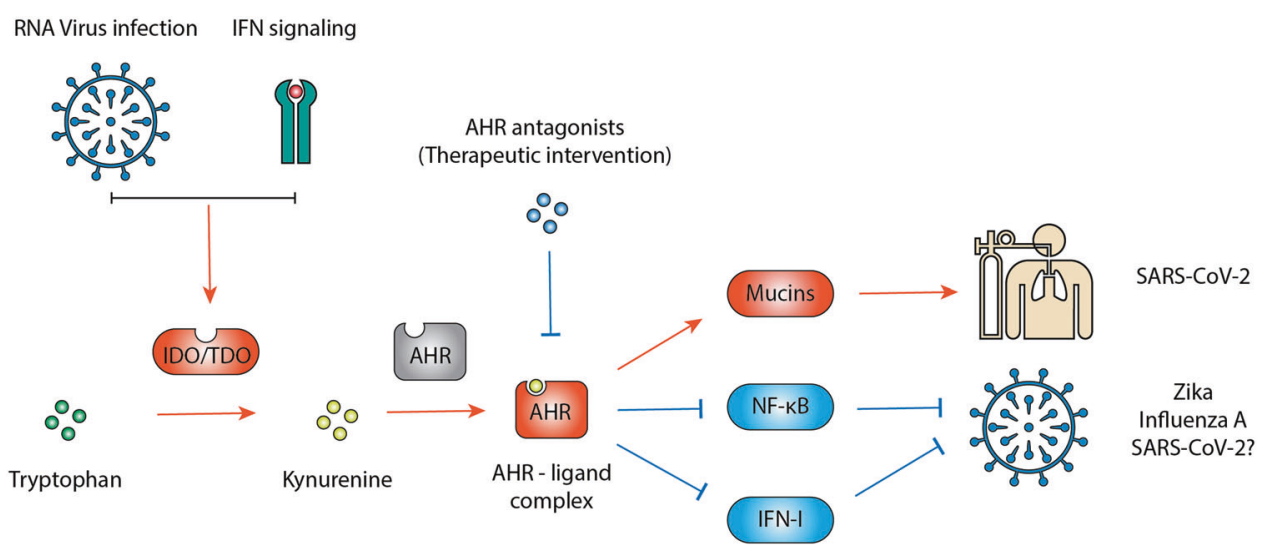

Fig. 1 AHR is a candidate therapeutic target for viral infection. AHR activation during viral infection results in the upregulation of IDO/TDO, which convert tryptophan to Kynurenine (Kyn). Kyn activates AHR, leading to formation of an AHR-ligand complex that limits host anti-viral responses mediated by IFN-I and NF- $\kappa B$, thus promoting viral replication. AHR signaling also induces mucin expression in lung epithelial cells, thickening the blood-air barrier, impairing $\mathrm{O}_{2}$ diffusion and causing hypoxia. AHR antagonists limit AHR activation, boosting the host anti-viral response and consequently reducing viral replication. AHR antagonism also reduces the expression of mucins, limiting lung pathology during SARS-CoV-2 infection.

identifying AHR as a candidate target for novel anti-viral therapies. The work by Liu et al. highlights the need to characterize the role of AHR in virus-induced pathology and the mechanisms involved, to guide the development of AHR-targeted therapies for virusinduced diseases.

\section{ADDITIONAL INFORMATION}

Competing interests: F.J.Q. is a member of the Scientific Advisory Board of Kyn Therapeutics.

\section{REFERENCES}

1. Huang, C. et al. Lancet 395, 497-506 (2020).

2. Tobin, M. J. et al. Am. J. Respir. Crit. Care Med. 202, 356-360 (2020).

3. Liu, Y. et al. Cell Res. https://doi.org/10.1038/s41422-020-00435-z (2020).

4. He, J. et al. Protein Cell 11, 680-687 (2020).

5. Bian, X. W. et al. Natl. Sci. Rev. 7, 1414-1418 (2020).

6. Gutierrez-Vazquez, C. et al. Immunity 48, 19-33 (2018).

7. Rothhammer, V. et al. Nat. Med. 22, 586-597 (2016).

8. Yamada, T. et al. Nat. Immunol. 17, 687-694 (2016).

9. Giovannoni, F. et al. Nat. Neurosci. 23, 939-951 (2020).

10. Giovannoni, F. et al. Res. Sq. https://doi.org/10.21203/rs.3.rs-25639/v1 (2020). 